\title{
Role of Influenza in ED Visits and Hospitalizations of Adults Over 65 Years in France
}

\author{
Vanina Bousquet ${ }^{\star 1}$, Larissa Vernier ${ }^{1}$, Yann Le Strat ${ }^{1}$, Isabelle Bonmarin ${ }^{1}$, Christophe \\ Leroy $^{2}$, Maurice Raphaël ${ }^{3}$, Gilles Viudes ${ }^{5}$, André de Caffarellii and Céline Caserio- \\ Schönemann ${ }^{1}$
}

${ }^{1}$ French Institute for Public Health Surveillance, St Maurice, France; ' 2 ouis Mourier Hospital Emergency Department, Colombes, France; ${ }^{3}$ French Society of Emergency Medicine, Paris, France; ${ }^{4}$ Bastia Hospital Emergency Department, Bastia, France; ${ }^{5}$ Federation of Regional Observatories for Emergency Activities, Paris, France

\section{Objective}

To estimate the real burden of influenza epidemic on emergency departments (ED) attendances and hospitalizations among patients over 65 years in order to better understand determinants of overcrowding and mortality excess.

\section{Introduction}

While the link between excess winter mortality and winter respiratory diseases in the elderly is well described, the impact of the epidemic of influenza in the elderly is mainly assessed in France through specific surveillance in the general population. Syndromic surveillance data enables to monitor ED attendances and hospitalizations for various diagnostic codes groupings throughout the influenza epidemic, some of which often cited as influenza proxies, such as cardiorespiratory diagnostic groups ${ }^{1-2}$.

In mainland France, the 2014-15 season was characterized by an intense influenza epidemic in the community (sub-type A(H3N2) dominant virus). Hospital overcrowding was early reported, partly linked to serious clinical presentations among the elderly, and leading to the triggering of a national emergency plan.

We hypothesized that ED numbers of clinical influenza cases underestimate the influenza burden among patients aged 65 years and over, especially when a $\mathrm{A}(\mathrm{H} 3 \mathrm{~N} 2)$ influenza subtype circulates.

\section{Methods}

In October 2014, the French syndromic surveillance system based on the ED (OSCOUR ${ }^{\circledR}$ network) included about 550 ED $(45,000$ daily attendances), recording $85 \%$ of the national total attendances. About 270 ED transmitted daily during the whole study period (20,500 daily attendances).

Numbers of visits and hospitalizations for influenza proxy variables were extracted from the national database for the period October 2010 to April 2015 and aggregated to a weekly level for patients aged 65 years and over. Weekly isolates of influenza viral subtypes were obtained from reference laboratories. Diagnostic codes groupings were selected based on their association with either influenza symptoms or complications of an influenza infection. Visual examination of times series correlation with influenza positivity rate along with spearman correlation were performed to select principal influenza proxy variables.

Attendances and hospitalizations of patients aged 65 years and over were modeled separately as a function of each selected proxy variables for influenza, seasonality and trend using a negative binomial regression model $^{2}$.

Two surrogate's measures for influenza activity were tested: clinical influenza attendances and laboratory confirmed influenza identifications (positivity rate). Numbers of visits for bronchiolitis among children under 2 years old were used as a proxy of RSV activity.

\section{Results}

For the entire study period, visits were significantly associated with influenza positivity rate for acute bronchitis (IRR $=2.095 \%$ $\mathrm{CI}=1.7 ; 2.3$ ), pneumonia (IRR $=1.495 \% \mathrm{CI}=1.2 ; 1.5)$, chronic obstructive pulmonary disease (COPD) $(\mathrm{IRR}=1.495 \% \mathrm{CI}=1.3 ; 1.6)$, dyspnea $\quad(\mathrm{IRR}=1.295 \% \mathrm{CI}=1.2 ; 1.3)$, asthma (IRR $=1.595 \%$ $\mathrm{CI}=1.3 ; 1.8$ ), acute cardiac failure (IRR $=1.295 \% \mathrm{CI}=1.1 ; 1.3)$, and dehydration $(\mathrm{IRR}=1.295 \% \mathrm{CI}=1.1 ; 1.4)$. Similar measures of associations were found for hospitalizations.

\section{Conclusions}

Our study shows that the burden of influenza is underestimated among the elderly, due to the lack of sensitivity of coding, especially during $\mathrm{A}(\mathrm{H} 3 \mathrm{~N} 2)$ seasons, partly explained by the large panel of influenza clinical presentations in this age group.

Based on our results, a diagnostic codes grouping will be specifically built to monitor influenza epidemic in ED and to estimate its burden among patients aged 65 years and over.

\section{Keywords}

influenza; emergency departments; flu proxy variables

\section{References}

1. Schanzer DL, Langley JM, Tam TWS. Hospitalization attributable to influenza and other viral respiratory illnesses in Canadian children. Pediatr Infect Dis J. sept 2006;25(9):795-800.

2. Thompson WW, Shay DK, Weintraub E, Brammer L, Bridges CB, Cox NJ, et al. Influenza-associated hospitalizations in the United States. JAMA. 15 sept 2004;292(11):1333-40.

\section{*Vanina Bousquet}

E-mail: v.bousquet@invs.sante.fr 